\title{
Craniotabes in a newborn
}

\author{
Yuko Wada MD, Katsuyuki Kubo MD, Shinichi Tsubata MD
}

U Cite as: CMAJ 2020 October 5;192:E1163. doi: 10.1503/cmaj.200192

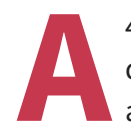

40-year-old woman who was G2P2 delivered a full-term male infant after an uncomplicated prenatal course. On physical examination of the newborn, we could not palpate the cranial bones at the top of his head. His extremities and facial appearance showed no abnormalities that would suggest syndromic malformation. Cranial computed tomography showed extensive areas of delayed ossification along the sagittal suture, and multiple small lesions in the temporal and occipital bones, which we did not detect on physical examination (Figure $1 A$ and $B$ ).

Laboratory tests performed on the neonate's fifth day of life showed the following results: serum 25-hydroxyvitamin $D$ level of 14.1 (normal range $20-100$ ) $\mathrm{ng} / \mathrm{mL}$ ) and increased parathyroid hormone level of 91 (normal range 10-60) pg/mL). We diagnosed vitamin $\mathrm{D}$ deficiency and secondary hyperparathyroidism. Radiographs of his extremities did not show evidence of rickets. His mother had no history of syphilis nor had she restricted her diet or sun exposure. However, her serum 25-hydroxyvitamin D level was low (9.4 [normal range, 30-100] ng/mL). We prescribed normal feeding with vitamin $D$ supplementation, which resulted in improved serum vitamin D levels. The infant's cranial bones hardened at 2 months of age, and he has shown normal development thereafter.

The incidence of craniotabes, defined as a softening of the skull bones, ranges from $20 \%$ to $30 \%$ internationally (although severe cases like we describe here are rare) and is directly correlated with maternal vitamin D levels. ${ }^{1}$ With adequate nutrition, it resolves spontaneously within 2-3 months. ${ }^{1}$ Craniotabes is also associated with pathological conditions, such as rickets, congenital syphilis or skeletal dysplasias. ${ }^{1}$ Our case was most likely due to maternal vitamin D deficiency. The prevalence of vitamin D deficiency in pregnant woman ranges from 50\% to $70 \%$ in developed countries, depending on geographic location. ${ }^{2}$

The American College of Obstetricians and Gynecologists recommends that pregnant women receive $600 \mathrm{IU} /$ day of supplemental vitamin D; however, 1000-2000 IU/day may be needed if vitamin $\mathrm{D}$ deficiency is identified. ${ }^{3}$

\section{References}

1. Yorifuji J, Yorifuji T, Tachibana K, et al. Craniotabes in normal newborns: the earliest sign of subclinical vitamin D deficiency. J Clin Endocrinol Metab 2008;93:1784-8.

2. Saraf R, Morton SM, Camargo CA Jr, et al. Global summary of maternal and newborn vitamin D status - a systematic review. Matern Child Nutr 2016;12:647-68.

3. ACOG Committee on Obstetric Practice. ACOG Committee Opinion No. 495: Vitamin D: screening and supplementation during pregnancy. Obstet Gynecol 2011;118:197-8 [reaffirmed 2017].

\section{Competing interests: None declared.}

This article has been peer reviewed.

The authors have obtained parental consent.

Affiliation: Department of Pediatrics, Toyama Red Cross Hospital, Toyama, Japan

Acknowledgement: The authors thank Dr. Neil Bowles for his assistance with the editing of the manuscript.

Correspondence to: Yuko Wada, ykwada22@gmail.com 